\title{
Neues vom Arznei- und Gewürzpflanzenanbau
} aus Bernburg

Das 27. Bernburger Winterseminar für Arznei- und Gewürzpflanzen bot wie in den vergangenen Jahren ein produktives Diskussionsforum zwischen Anbauern, Herstellern von pflanzlichen Produkten und der Wissenschaft. Mehr als 280 Teilnehmer kamen am 21. und 22. Februar 2017 nach Bernburg, was eine deutliche Steigerung gegenüber den vergangenen Jahren darstellte und die Kapazität der Bernburger Hotels leicht überforderte aber schließlich fand jeder im näheren Umland ein Bett für die Nacht. Wie immer wurde die Tagung von Saluplanta e. V. organisiert; an dieser Stelle sei schon einmal Herrn Bernd Hoppe herzlich gedankt.

\section{Immer wieder aktuell: NEM}

Eröffnet wurde die Fachtagung durch Prof. Dr. Falko Holz von der LLG Bernburg, der die Grüße der Ministerin für Landwirtschaft und Umwelt, Frau Prof. Dr. Claudia Dalbert, überbrachte. Der erste Tag war geprägt von eher allgemeinen und regulatorischen Themen. Im Eröffnungsvortrag stellte Dr. Nicole Armbrüster vom BPI Berlin die aktuellen Entwicklungen auf dem Gebiet der „Health Claims“ für Nahrungsergänzungsmittel (NEM) vor. Allein in Deutschland gibt es jedes Jahr ca. 5000 neue NEM. Das Problem: Durch den leichten Marktzugang steigt der Anreiz, Fertigprodukte mit Heilpflanzen als NEM auf den Markt zu bringen und eben nicht als Phytopharmakon. Frau Dr. Armbrüster wird die derzeitige Situation in einem eigenen Beitrag in einer der kommenden Ausgaben der ZPT vorstellen.

Mit NEM befasste sich auch der Vortrag von Prof. Dr. Chlodwig Franz, Uni-

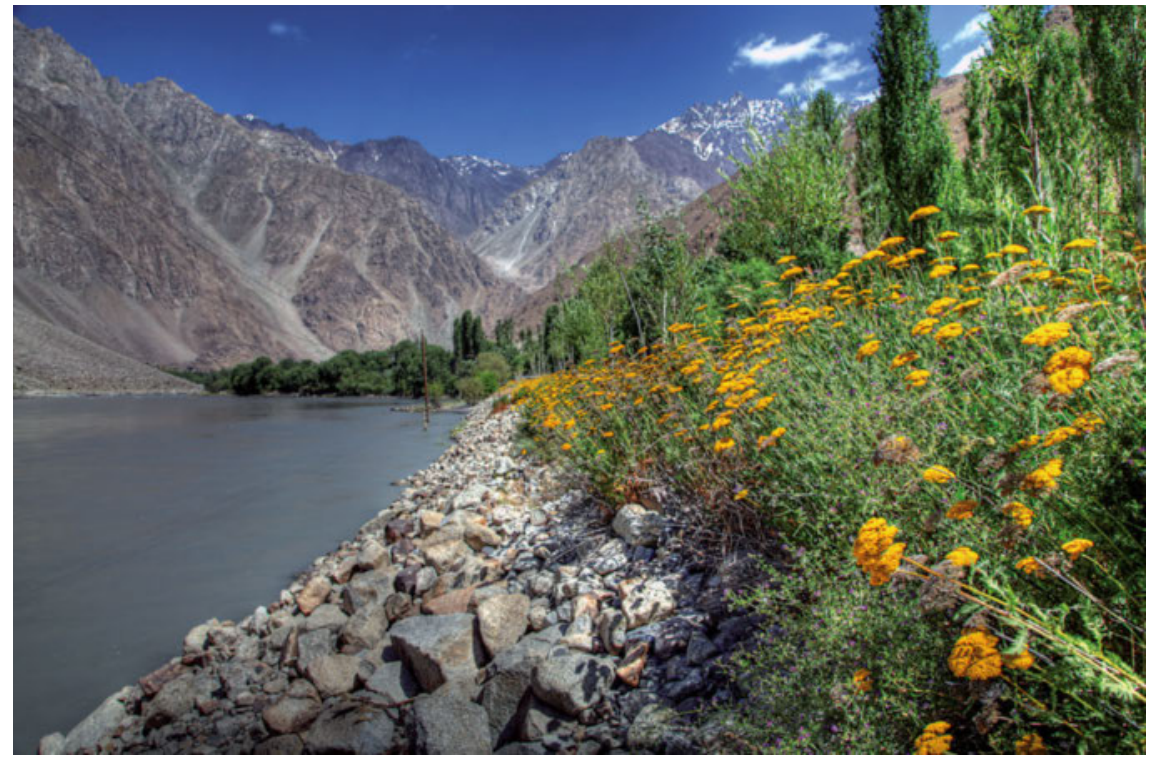

- Abb. 1 Dies ist nicht Bernburg, sondern der Panj-Fluss, der die Grenze zwischen Afghanistan (links) und Tadschikistan (rechtes Ufer) bildet. Die u. a. dort heimische gelb blühende Schafgarbe wird neuerdings in Hessen angebaut. (c) Prof. Keusgen

versität Wien. Prof. Franz bemängelt innerhalb des EU-geförderten PlantLIBRAProjektes die oftmals anzutreffenden Kontaminanten. Franz weist auf den Paradigmenwechsel im Nahrungsmittelbereich hin: Bis in die 1970er Jahre wurden „Anti-Nutritive“ abgereichert, danach wurde der Fokus immer mehr auf pflanzliche Sekundärstoffe gelegt, die einen gesundheitlichen „Mehrwert“ in sich bergen. Viele dieser Stoffe werden zwar im Darm metabolisiert, aber die Umwandlungsprodukte werden dann doch deutlich resorbiert. Zudem können pflanzliche Sekundärmetabolite die Resorption von Kohlenhydraten im Darm behindern, was einer antidiabetischen Wirkung entsprechen würde.

\section{Beikräuter als Kontaminanten}

Nachfolgend referierte Dr. Barbara Steinhoff vom BAH in Bonn über den aktuellen Stand der Diskussion über die Pyrrolizidinalkaloide (PA). Derzeit gibt es einen „Code of Practice“ zur Reduktion der PA-Belastung und eine Datenbank zur PA-Belastung wird unter dem Dach der Verbände aufgebaut. Ferner soll eine standort- und kulturpflanzenspezifische Beikrautflora erhoben und die Toxizität einzelner Pyrrolizidinalkaloide bewertet werden. Auch zu diesem Thema wird es einen separaten Beitrag in der ZPT geben. Ebenfalls steht derzeit die Miternte von Fremdpflanzen im Fokus, z. B. Solanaceen und Convolvulaceen, die zu Funden von Tropanalkaloiden im Erntematerial führen. Dieses ist für Säuglinge und 


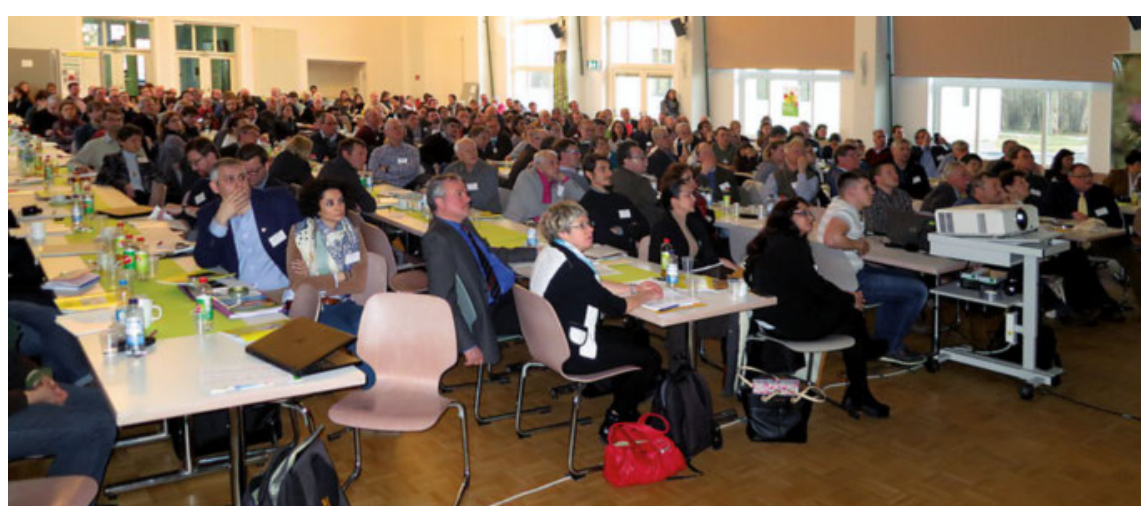

- Abb. 2 Etwa 280 aufmerksame Zuhörer fanden sich zum diesjährigen Winterseminar ein. (C) Prof. Keusgen

Kleinkinder von Relevanz. So gibt es in $70 \%$ der Kräutertees positive Befunde.

Dr. Maik Kleinwächter von der TU Braunschweig erörterte, dass PAs, ähnlich wie Nikotin, auch über die Wurzel aufgenommen werden können („horizontaler Naturstoff-Transfer“). Hierzu wurden Anbauversuche auf Mulch von JakobsKreuzkraut (Senecio jacobaea) durchgeführt. Es ließen sich Werte oberhalb $50 \mu \mathrm{g} / \mathrm{kg}$ Trockengewicht erzielen, was als bedenklich eingestuft werden kann. Überschlägig gerechnet, wurde ca. $1 \%$ der Kreuzkraut-PAs aus dem Test-Mulch über die Wurzel der Kulturpflanze aufgenommen. Besonders empfänglich für exogene PAs zeigte sich Petersilie. Hierbei sei jedoch angemerkt, dass der verwendete Mulch sehr PA-reich war und dieses experimentelle „Setting“ so wohl kaum im Anbau vorkommt. Als praktische Konsequenz sollten PA-haltige Beikräuter nicht nur ausgerissen, sondern vollständig vom Acker entfernt werden. Nicht beantwortet werden konnte die Frage, mit welchem PA-Eintrag über Kompost zu rechnen ist.

Das Thema Pestizidrückstände aus Pflanzenschutzmitteln (PSM) wurde in einem Vortrag von Dr. Bernhard Klier, Phytolab Vestenbergsgreuth, vertieft. Rückstandshöchstgehalte sind innerhalb Europas einheitlich geregelt. Jedoch werden häufig geringe Mengen PSM gefunden, die gar nicht auf das Feld aufgebracht wurden. Hier stellt sich die Frage nach den Ursachen. Dieses erfordert insbesondere eine validierte Analytik; falschnegative sowie falschpositive Ergebnisse sind möglich, wie beispielsweise angebliche
PSM bei Ingwer. Häufig treten Ergebnisse im Bereich der Bestimmungsgrenze auf, die mit nachgeschalteter Analytik abzusichern seien.

\section{Cannabis}

Prof. Dr. Michael Keusgen, Universität Marburg, stellte die aktuelle Entwicklung von Medizinalhanf (Cannabis sativa) vor. Hanfblüten können seit März 2017 auf BTM-Rezept verordnet werden, woraus sich die Frage ergibt, woher denn nun das Material kommen soll. Zu diesem Zweck wird am BfArM eine Cannabisagentur eingerichtet (Leitung: Prof. Dr. Werner Knöss), über die dann Medizinalhanf eingekauft wird und an den pharmazeutischen Großhandel oder direkt an Apotheken weitergegeben werden kann. Das Blütenmaterial muss der neuen Arzneibuchmonografie Cannabisblüten entsprechen, wobei $\Delta 9$-Tetrahydrocannabinol, Cannabidiol und die korrespondierenden Säuren bestimmt werden. Problematisch erscheint derzeit die Haltbarkeit der Cannabisblüten. Bei Raumtemperatur ist die Droge wohl nur 6 Monate haltbar. Für Cannabidiol gibt es ermutigende Ergebnisse in der Krebstherapie; leider unterliegt die Substanz seit kurzem wegen möglicher Arzneistoffinteraktionen der Verschreibungspflicht. Insgesamt ist mit einer starken Belebung der CannabisNachfrage zu rechnen, jedoch sind Cannabinoide auch synthetisch zugänglich. Im Gegensatz zu einigen Bundesstaaten in USA ist der Markt in Europa streng reguliert, wodurch allzu große Erwartungen wohl gedämpft werden müssen.

\section{Russland}

Im weiteren Tagungsverlauf wurde der Arzneipflanzensektor in ausgewählten Ländern vorgestellt. Prof. Dr. Elena Malankina aus Moskau erörterte den Markt in Russland. Das derzeitige Marktvolumen für Arzneimittel insgesamt beträgt ca. 13 Mrd. Euro. Pflanzliche Arzneimittel machen jedoch nur 2\% des Marktvolumens aus. Übliche Arzneitees sind z.B. Hagebutte, Salbei oder Brennnessel. Nach Indikation haben Expektorantien, Sedativa (hierzu gehören auch Baldrian und Pfingstrose Paeonia spec.) sowie Tonika (Adaptogene) den höchsten Marktanteil. Typischerweise kostet eine Packungseinheit pflanzliches Arzneimittel weniger als einen Euro, was die Frage nach dem angewandten Qualitätsstandard aufwirft. Als Fertigarzneimittel sind Baldrian und Weißdorn am gebräuchlichsten. Die Gesamternte beträgt jährlich etwa $17000 \mathrm{t}$, zumeist über Wildsammlungen. Arzneipflanzen werden punktuell angebaut, vorwiegend im Süden des Landes. Auch Sibirien scheint eine reiche Schatzkammer für Arzneipflanzen zu sein. Frau Malankina vertritt die These, dass dort die natürlichen Ressourcen nahezu unerschöpflich, aber nur schwer zugänglich sind.

\section{Türkei}

Irfan Evcin aus Yalova schildert die Situation in der Türkei. In großem Stil werden Gewürzpflanzen wie Oregano, Lorbeer, Anis, Fenchel und Kreuzkümmel angebaut. 1994 kam die Pfefferminze dazu. Der Anbau von „reinen“ Arzneipflanzen wie z.B. Johanniskraut nimmt eine untergeordnete Stelle ein. Der Export an Gewürzpflanzen liegt über $35000 t$ jährlich. Als Einzelprojekt wird das Sammeln von Wildapfel vorgestellt. Ferner gehört die Türkei zu den weltweit größten Produzenten von Rosenöl.

\section{Albanien}

Dr. Nazim Gruda von der Universität Bonn stellt den Arzneipflanzenmarkt in Albanien vor. Hier werden mehr als 300 Wildarten arzneilich genutzt, die zumeist gesammelt werden. Insbesondere 
sind Oregano, Winter-Bohnenkraut (Satureja montana), Thymian, Johanniskraut, Melisse, Salbei, Wachholder und Weißdorn von Bedeutung. Derzeit werden ca. 15000t Arznei- und Gewürzpflanzen exportiert. Besonders wichtig für den Export ist Salbei, gefolgt von Oregano und Winter-Bohnenkraut. Beim Anbau wurden das minderwertige Saatgut und der geringe Mechanisierungsgrad als Problem aufgeführt. Ungelöst sind Fragen des Naturschutzes, des ökologischen Anbaus und die Rückverfolgbarkeit des gesammelten Materials.

\section{Aspekte der Pflanzenzüchtung und des Pflanzenschutzes}

Dr. Elke Bloem, JKI Braunschweig, berichtete über die Stressinduktion bei Arznei- und Gewürzpflanzen. Die Untersuchungen wurden an mehreren, recht unterschiedlichen Arzneipflanzen wie Johanniskraut, Kapuzinerkresse, Schöllkraut, Thymian und Petersilie durchgeführt. Der „Versuchsstress“ bestand aus leichter Trockenheit und erhöhtem Salzgehalt sowie aus den Phytohormonen Methyljasmonat und Salicylat. Zusätzlich wurde Zelllysat als Spray angewandt; dieses sollte Insektenfraß simulieren. Dabei führte Trockenstress zumeist zu einer Erhöhung der sekundären Metabolite, jedoch reduzierte sich erwartungsgemäß die Biomasse. Bessere Ergebnisse wurden mit Methyljasmonat erzielt. Salycilatbehandlung führte beim Johanniskraut zu einem erhöhten Hypericingehalt, verbunden mit einer größeren Biomasse. Insgesamt waren die Ergebnisse aber eher uneinheitlich.

Dr. Wolfram Junghanns, Aschersleben, erklärt eine neue Rosmarinus-officinalisZüchtung, die auf Carnosolsäure standardisiert ist. Bekanntester Inhaltsstoff des Rosmarins ist die Rosmarinsäure, aber auch die Carnosolsäure ist von Bedeutung. Beides sind natürliche Antioxidantien. Insbesondere mit Carnosolsäure können eher fettige Zubereitungen (Kosmetika) länger haltbar gemacht werden. Die Carnosolvarietät des Rosmarins wurde innerhalb von 15 Jahren aus unterschiedlichen Herkünften wie Portugal,

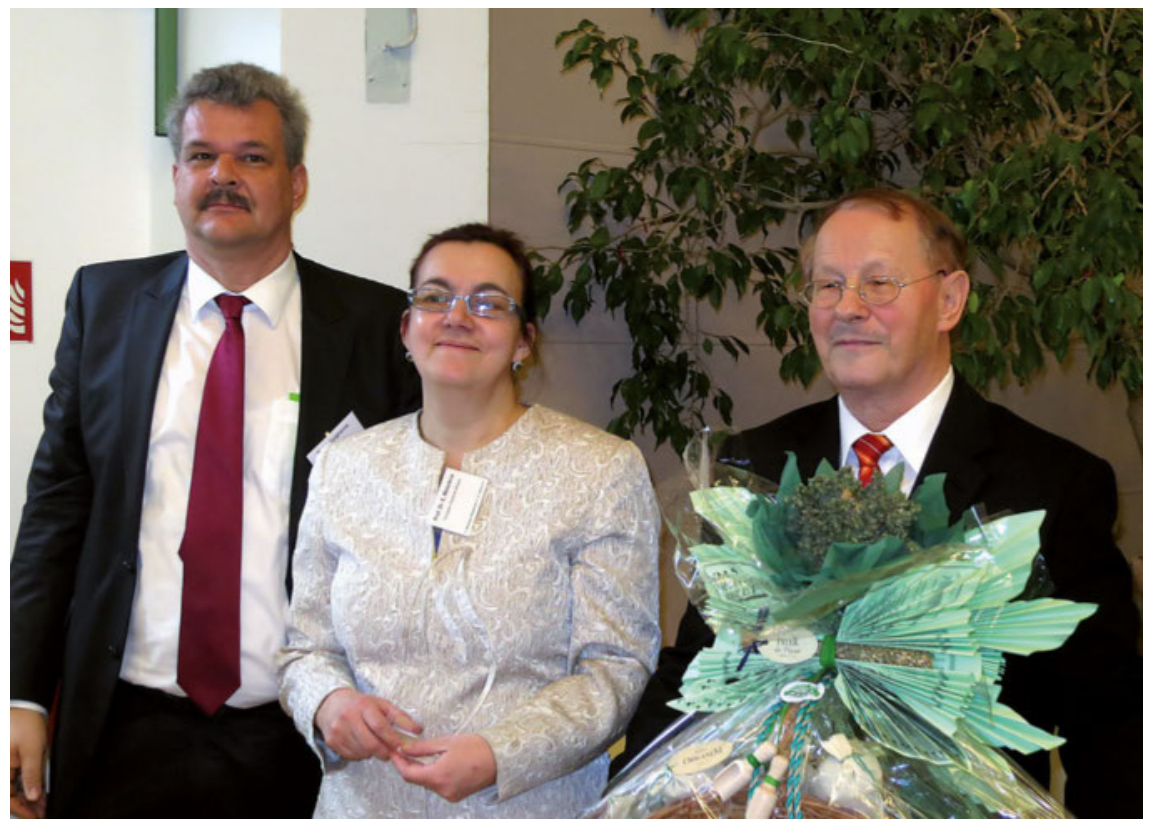

- Abb. 3 Preisübergabe an Frau Prof. Malankina durch Herrn Dr. Junghanns (links) und Herrn Hoppe (rechts). @ Prof. Keusgen

Spanien, Griechenland und dem Gebiet des ehemaligen Jugoslawiens herausselektiert. Es konnte ein Gehalt von mindestens $10 \%$ Carnosolsäure erreicht werden; derzeit steht die optimierte Züchtung auf 10 Hektar. Das getrocknete und feinvermahlene Material kann ohne weitere Extraktion zur Konservierung eingesetzt werden.

Eberhardt Walther, Landesbetrieb Landwirtschaft Hessen, stellte einen Anbau der gelb blühenden Schafgarbe Achillea filipendulina („Goldgarbe“) in Nordhessen vor. Der ca. 3 Hektar umfassende Anbau ist eine Besonderheit, da diese Art ursprünglich aus dem Kaukasus und Mittelasien stammt ( $\triangleright$ Abb. 1); dort wird sie volksmedizinisch verwendet. Wegen ihrer Herkunft wächst die Art nicht auf staunassen und schattigen Standorten; der Boden sollte sonnig, nährstoffreich und ausreichend feucht sein. Geerntet werden die blühenden Sprossen. Da die Pflanze mehrjährig ist, kann über mehrere Jahre geerntete werden, wobei bei älteren Pflanzen der Ertrag nachlässt. Der Ertrag beträgt 2,5-3t/Hektar. Auch wenn es sich um eine potenzielle Arzneipflanze handelt, wird das Pflanzenmaterial nahezu ausschließlich für floristische Zwecke verwendet. Nach maximal 12 Jahren muss das Feld erneuert werden.

\section{Ehrungen}

Innerhalb der Tagung fanden auch zwei Ehrungen statt. Der Saluplanta-Ehrenpreis ging an Prof. Dr. Wolf-Dieter Blüthner für seine Grundlagenforschung an der Universität Halle sowie für Züchtungen an Majoran, Johanniskraut und Thymian. Daneben war Herr Blüthner auch sehr erfolgreich an der Züchtung von Zierpflanzen beteiligt. Der GFS-Ehrenpreis ging an Prof. Dr. Elena Malankina ( $\triangleright$ Abb. 3) aus Moskau für die Erforschung und Inkulturnahme von Lamiaceen wie z.B. dem Drachenkopf.

\section{Fazit}

Das Bernburger Winterseminar hat einmal mehr gezeigt, dass regulatorische Aspekte sowie die Betrachtung von Kontaminanten einen immer breiteren Raum beim Anbau von Arzneipflanzen einnehmen. Hier konnte die Tagung sehr zum Verständnis dieser komplexen Materie beitragen und den Dialog zwischen Anbauern und Herstellern in konstruktiver Weise unterstützen. Alle sind herzlich zum 28. Bernburger Winterseminar im Februar 2018 eingeladen.

Prof. Dr. Michael Keusgen, Marburg 